'Dermatólogo, Departamento de Dermatología, Facultad de Medicina, Pontificia Universidad Católica de Chile. Santiago, Chile. ${ }^{2}$ Residente de Dermatología, Departamento de Dermatología, Facultad de Medicina, Pontificia Universidad Católica de Chile. Santiago, Chile.

aInterna de Medicina, Pontificia Universidad Católica de Chile. Santiago, Chile.

Fuente de apoyo financiero: No hubo. Recibido el 10 de agosto de 2015, aceptado el 19 de abril de 2016.

Correspondencia a: Dr. Néstor Carreño Orellana Departamento de Dermatología, Facultad de Medicina, Pontificia Universidad Católica de Chile. Av. Vicuña Mackenna 4686, Macul. Santiago, Chile. Teléfono: (56 2) 23548659 Fax: (56 2) 25529974. nestorcarreno@yahoo.es

\section{Efectos adversos de finasteride: mitos y realidades. Una revisión actualizada}

\author{
NÉSTOR CARREÑO-ORELLANA ${ }^{1}$, CATHERINA MOLL-MANZUR ${ }^{a}$, \\ JUAN EDUARDO CARRASCO-ZUBER ${ }^{2}$, SERGIO ÁLVAREZ-VÉLIZ', \\ DANIELABERROETA-MAURIZIANO ${ }^{3}$, NINOSKAPORRAS-KUSMANIC ${ }^{2}$
}

\section{Finasteride adverse effects. An update}

Finasteride is a 5- $\alpha$ reductase inhibitor that is widely used in the management of benign prostate hyperplasia and male pattern hair loss. It is well known that these agents improve the quality of life in men suffering from these conditions. However, they are associated with some transient and even permanent adverse effects. The aim of this article is to clarify the controversies about the safety of finasteride by analyzing the evidence available in the literature.

(Rev Med Chile 2016; 144: 1584-1590)

Key words: Alopecia; Drug-Related Side Effects and Adverse Reactions; Finasteride; Therapeutics.
F inasteride es un fármaco, cuyo mecanismo de acción se basa en la inhibición competitiva selectiva de la enzima $5-\alpha$ reductasa tipo 2. Esto impide la conversión de testosterona a su forma más potente, la dihidrotestosterona (DHT), reduciendo así marcadamente los niveles plasmáticos de esta hormona. Se ha visto, que tan sólo una dosis de $1 \mathrm{mg} /$ día, logra una reducción de DHT entre un $60 \%$ y $70 \% 0^{1,2}$.

En 1992 se aprobó el uso de finasteride a una dosis de $5 \mathrm{mg}$ /día para el tratamiento de la hiperplasia prostática benigna (HPB). Posteriormente, en 1997 se extendió su uso a dosis de $1 \mathrm{mg} /$ día para tratar la alopecia androgénica (AGA) en hombres. Desde entonces, finasteride ha sido utilizado exitosamente en AGA. Esta nueva indicación del uso de finasteride ha traído como consecuencia su uso en etapas más precoces y prolongadas de la vida, por lo que se han reportado nuevos efectos adversos de su uso.

En este artículo presentamos una revisión acerca de los efectos adversos del tratamiento con finasteride, principalmente en relación a su uso en
AGA, con el objetivo de aclarar las controversias existentes en relación a este tema.

\section{Cáncer de mama}

Dado que entre los años 1992 y 2009 hubo 50 casos reportados de cáncer de mama en pacientes que usaban finasteride a dosis de $5 \mathrm{mg} / \mathrm{día}^{3}$, la Food and Drug Administration (FDA) en el año 2009 publicó una advertencia en cuanto al posible mayor riesgo de desarrollar cáncer de mama en pacientes usuarios de finasteride.

Evidencia de la asociación entre finasteride y cáncer de mama en hombres viene del estudio randomizado, doble ciego y placebo controlado, llamado "Medical Therapy of Prostatic Symptoms" (MTOPS) ${ }^{4}$. En este estudio, que incluyó a 3.047 hombres, sólo hubo 4 casos de cáncer de mama, todos asociados al uso de inhibidores de 5- $\alpha$ reductasa (5-IAR) a dosis para HBP. Considerando la baja incidencia de cáncer de mama en hombres, esto significó un riesgo 200 veces mayor 
de desarollar cáncer de mama versus la población general.

Sin embargo, en otros dos estudios estudios clínicos randomizados: "The Proscar Long-Term Efficacy and Safety study" (PLESS) $)^{5}$ con seguimiento a 4 años y "Prostate Cancer Prevention Trial” (PCPT) ${ }^{6}$ con seguimiento a 7 años, no se encontró mayor incidencia de cáncer de mama en el grupo tratado con finasteride versus el grupo placebo.

Los autores de un reciente estudio de cohorte, encontraron que los hombres que usaron 5-IAR no tuvieron mayor riesgo de desarrollar cáncer de mama, en cambio los que fueron manejados con resección transuretral para su HBP, sí presentaron mayor riesgo en relación a hombres que no habían sido expuestos a ninguno de estos dos tratamientos. Por ello, sugirieron que el medio endocrinológico, que conduce al HBP, podría aumentar el riesgo de cáncer de mama y no el fármaco en sí mismo, considerando que la resección transuretral se usa en pacientes con síntomas más severos y próstatas más grandes ${ }^{7}$.

El mecanismo, por el cual finasteride podría causar cáncer de mama en hombres, sería el desbalance entre los niveles de estrógenos y andrógenos ${ }^{7,8}$, que se produciría a consecuencia de la disminución de DHT y del aumento de los niveles de testosterona.

Se ha postulado, un posible efecto mutagénico de los estrógenos, debido a que se ha visto que aumentan la actividad de telomerasas, que inducen división celular y con ello, podrían originar neoplasias.

En resumen, la evidencia disponible en cuanto al riesgo de desarrollar cáncer de mama en hombres usuarios de finasteride es controversial. En cuanto a este efecto en mujeres, no existen datos en la literatura. Sin embargo, dada la observación de un posible aumento del riesgo de cáncer de mama en los hombres, su uso off-label sólo debe hacerse en mujeres con examen físico normal y sin antecedente personal de cáncer de mama.

\section{Cáncer de próstata}

La mejor evidencia disponible en cuanto a este tema, se encuentra en los resultados que mostró el estudio $\mathrm{PCPT}^{9}$. Se concluyó, que el uso de finasteride a dosis de $5 \mathrm{mg} /$ día versus placebo está asociado a una reducción del riesgo relativo de un 30\% de desarrollar cáncer de próstata (RR: 0,7, IC 95\%: 0,65-0,76, p < 0,001), a 10 años de seguimiento en hombres mayores de 54 años de edad, con un tacto rectal inicialmente normal y un antígeno prostático específico inicial menor a 3,0 $\mathrm{ng} / \mathrm{mL}$. Al estratificar por grado, se encontró que el uso de este fármaco confiere protección (RR: $0,57(0,52-0,63) \mathrm{p}<0,001)$ contra el desarrollo de cáncer de próstata de bajo grado, medido por un score de Gleason de 2-6. En cambio, el riesgo relativo de desarrollar un cáncer prostático de alto grado (Gleason 7-10) tendió a ser mayor en el grupo tratado con finasteride (RR: 1,17, IC 95\%: $1,00-1,37, p=0,05)$, pero no hubo diferencias estadísticamente significativas en términos de mortalidad a 10 años.

Resultados similares se evidenciaron en el estudio llamado "Reduction by Dutasteride of Prostate Cancer Events (REDUCE) trial ${ }^{10}$, que comparó otro 5-IAR (dutasteride) versus placebo.

El uso de dosis menores de finasteride ( $1 \mathrm{mg} /$ día) no ha reportado efectos protectores.

Desde el punto de vista fisiopatológico, no se ha demostrado que la testosterona o la 5- $\alpha \mathrm{DHT}$ causen la iniciación, promoción o desarrollo del cáncer de próstata ${ }^{11-13}$. Pese a que se ha postulado que la deprivación de andrógenos en el cáncer de próstata resulta inicialmente en la disminución del tamaño del tumor, se ha visto que luego de 18 a 24 meses, el cáncer se haría independiente de la cantidad de andrógenos circulantes y no requeriría de ellos para crecer ${ }^{11,12}$.

En resumen, dada la evidencia disponible, la FDA no ha aprobado el uso de 5-IAR en la quimio prevención del cáncer de próstata.

\section{Efectos adversos sexuales}

Diversos estudios clínicos randomizados ${ }^{4,6,14-17}$, doble ciego y placebo controlados, han encontrado que el uso de finasteride en dosis de $5 \mathrm{mg} /$ día aumenta el riesgo de sufrir experiencias sexuales adversas, las que incluyen: disfunción eréctil, disminución de la libido y disminución del volumen eyaculatorio, con riesgos absolutos estimados que varían entre $0 \%$ a $10 \%, 2 \%$ a $6 \%$ y $1 \%$ a $3 \%$ respectivamente ${ }^{18}$.

Aun cuando se ha visto que la incidencia de efectos sexuales no deseados con el uso de dosis 
altas de finasteride es mayor, no se ha encontrado un aumento en la tasa de discontinuación en los grupos tratados con finasteride versus placebo ${ }^{18}$.

Se ha sugerido el rol de factores psicológicos en la experiencia de efectos sexuales adversos asociados al uso de finasteride.

Mondaini y cols ${ }^{19}$, en su estudio prospectivo, simple ciego, estudió el efecto nocebo en pacientes a los cuales se les administró $5 \mathrm{mg}$ diarios de finasteride, encontrando que la incidencia de efectos adversos fue significativamente mayor en el grupo informado versus el no informado (43,6\% versus $14,3 \%)$. Más aún, los efectos adversos fueron completamente reversibles en 5 días posteriores a la suspensión del fármaco.

Por otro lado, en la literatura revisada se encontró sólo un estudio que evaluaba los efectos adversos sexuales al uso de finasteride concomitantemente con sus posibles factores confundentes. $\mathrm{El}$ análisis longitudinal del $\mathrm{PCPT}^{20}$ encontró que otras covariables, como diabetes, sedentarismo, hipertensión, tabaquismo, índice de masa corporal aumentado y la mayor edad, tenían efecto similar o incluso mayor que el uso de finasteride en la función sexual. Ninguno de los participantes reportó efectos sexuales permanentes en este estudio.

Los efectos sexuales, del uso de finasteride a dosis de $1 \mathrm{mg} /$ día, fueron evaluados en una revisión sistemática que incluyó ensayos clínicos randomizados. Se encontró, que el uso de finasteride versus placebo incrementa el riesgo de disfunción eréctil (RR: 2,22, IC 95\% 1,03-4,78; NNH: 82,1, IC 95\%: 56-231) con una calidad de evidencia moderada ( $\left.{ }^{2}: 1 \% ; \mathrm{p}: 0,41\right)$, sugiriendo que 1 de cada 80 pacientes experimenta disfunción eréctil. No se encontraron diferencias estadísticamente significativas en ambos grupos, con respecto a la disminución de la libido y los desórdenes de eyaculación. Tampoco se encontró diferencias estadísticamente significativas en las tasas de discontinuación por efectos adversos en ambos grupos $^{2}$.

Existen otros dos metaanálisis ${ }^{21,22}$; ambos no encontraron mayor riesgo de tener alteraciones sexuales secundarias al uso de 5-IAR, sin embargo, uno de ellos reportó un probable sesgo de detección en los resultados ${ }^{22}$.

Se ha discutido sobre la naturaleza persistente y la severidad de los efectos adversos sexuales asociados al uso de finasteride. Sin embargo, la evidencia existente es controversial.
Se ha visto, que la coadministración de tadalafil y finasteride mejora la disfunción sexual/ eréctil en pacientes mayores de 45 años con HPB sintomática a las 4, 12 y 26 semanas de uso, independiente de la presencia o no de disfunción sexual al comienzo del tratamiento. Por ello, en pacientes seleccionados que experimenten disfunción sexual atribuida a 5-IAR, que afecte su calidad de vida y en los cuales se decida no suspender el tratamiento por algún motivo, podría considerarse ese medicamento a menos que el paciente tenga contraindicaciones para su $\mathrm{uso}^{23}$.

\section{Alteraciones del espermiograma}

Se han encontrado alteraciones de las hormonas sexuales y de algunos parámetros del espermiograma asociados al uso de finasteride, tanto en pacientes sanos como en pacientes infértiles. Amory y cols. ${ }^{24}$, en su estudio multicéntrico randomizado, doble ciego, placebo controlado efectuado en pacientes sanos con parámetros de semen inicialmente normales, encontró que tras 1 año del uso de finasteride a dosis de $5 \mathrm{mg} /$ día, la DHT plasmática se redujo en un $72,7 \%$ bajo la línea basal ( $p<0,001)$, con un aumento de la testosterona plasmática en un $25 \%$, la que retornó a los niveles normales después de 4-12 semanas de la suspensión de la droga. A su vez, se encontró que la concentración de espermatozoides disminuyó en un $21,5 \%$, la motilidad de espermatozoides en un $10,5 \%$ y el volumen del semen en un $21,1 \%$ a las 26 semanas de tratamiento, hallazgos que resultaron estadísticamente significativos. El conteo de espermatozoides también experimentó una reducción significativa en un $34,3 \%$, sin embargo, sólo en un $5 \%$ de los pacientes está reducción era mayor al $10 \%$, sugiriendo una variabilidad individual de respuesta al fármaco. El único parámetro, que se mantuvo alterado a las 24 semanas posterior a la suspensión de finasteride, fue la reducción en la motilidad de los espermios. No hubo cambios significativos en la morfología de los espermios durante todo el período evaluado.

La mejor calidad de evidencia disponible sugiere, en cambio, que la dosis de $1 \mathrm{mg} /$ día de finasteride no ejerce efectos negativos sobre el espermiograma, sin embargo, sí se ha visto cambios en los parámetros hormonales ${ }^{25}$.

Un estudio en hombres infértiles, usuarios fi- 
nasteride en dosis bajas con una media acumulada de tratamiento de 57,4 meses, encontró un aumento estadísticamente significativo de 11,6 veces en el conteo basal de espermatozoides posterior a la suspensión de este fármaco, que fue mayor en los hombres con oligoespermia inicial severa. Sin embargo, concomitantemente se encontró que los niveles de la hormona folículo estimulante presentaron una tendencia hacia el límite superior del valor normal, lo que sugirió que estos hombres podrían haber tenido un nivel basal de subfertilidad. Los autores de este estudio sugirieron, que los hombres infértiles con azoospermia u oligoespermia suspendan el uso de finasteride, lo que podría mejorar la calidad del semen y facilitar alguna terapia de fertilidad ${ }^{26}$.

En cuanto a la patogenia, aún no se conocen con certeza los mecanismos por los cuales finasteride afecta mayormente la espermatogénesis en algunos hombres, sin embargo, se han planteado algunas hipótesis: 1) la existencia de una variabilidad individual de respuesta al fármaco, propuesto a partir del estudio de Amory y cols ${ }^{24}$; 2) una eventual disfunción epididimal secundaria a la reducción de la concentración de DHT propuesto a partir de estudios en $\operatorname{ratas}^{27}$; 3) un probable efecto negativo de finasteride sobre el ácido desoxirribonucleico de los espermios ${ }^{28}$; 4) la eventual presencia de un polimorfismo genético en el receptor de andrógenos en individuos con un espermiograma basal alterado, lo que se relacionaría a profundas alteraciones en el espermiograma al usar finasteride ${ }^{29}$.

\section{Efectos metabólicos}

\section{- Sobre el metabolismo óseo}

Los efectos del uso de los 5-IAR sobre el metabolismo óseo han sido medidos en pocos estudios y de baja calidad de evidencia. De acuerdo a ellos, los 5-IAR no ocasionarían una disminución de la densidad mineral ósea, ni una mayor predisposición a fracturas $\mathrm{s}^{30-33}$.

\footnotetext{
- Sobre el metabolismo de la glucosa y los lípidos

Las enzimas 5-a reductasa no sólo juegan un rol en el metabolismo de andrógenos, sino también en el de glucocorticoides, por lo que, se ha sugerido que su inhibición resultaría en un desbalance de ciertos procesos metabólicos ${ }^{34}$.
}

En este sentido, se ha visto que en pacientes obesos, la expresión de la enzima 5- $\alpha$ reductasa tipo 1 aumenta a mayor hiperinsulinemia y severidad de la esteatohepatitis no alcohólica, lo que sugiere su rol protector en la preservación de la sensibilidad hepática a la insulina ${ }^{35}$.

Un estudio doble ciego, randomizado y controlado, que evaluó los efectos metabólicos de finasteride y dutasteride en hombres con HPB sometidos a esta terapia por 3 meses, encontró que, en comparación con tamsulosina como control, la inhibición dual de las enzimas $5-\alpha$ reductasa tipo 1 y tipo 2 por Dutasteride disminuyó la sensibilidad a la insulina y aumentó la grasa corporal (no se menciona distribución), sin verse efectos significativos por la administración de finasteride ${ }^{36}$.

En relación con el efecto del uso de finasteride en altas y bajas dosis sobre el perfil de lípidos, hay estudios que evidencian que la alteración lipídica no es significativa en el corto ni largo

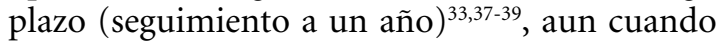
pueda existir un aumento inicial del colesterol HDL, LDL y total a los 3-4 meses, cuyos valores se normalizarían al año ${ }^{39}$.

Si bien es cierto que se ha visto que los 5-IAR podrían tener efectos deletéreos en el metabolismo de la glucosa y de los lípidos, no se conoce su impacto en términos de riesgo de diabetes mellitus y síndrome metabólico, con sus diferentes consecuencias adversas. En este sentido, se requieren estudios en hombres con HPB que evalúen a largo plazo el uso de los 5-IAR, sobre todo considerando que la misma HPB se ha asociado a síndrome metabólico ${ }^{40}$.

\section{Efectos cardiovasculares}

No se ha encontrado asociación estadísticamente significativa entre la inhibición de las 5-IAR y eventos cardiovasculares adversos ${ }^{41-43}$.

Por el contrario, estudios preclínicos efectuados en ratas, han sugerido un posible rol cardioprotector de finasteride ${ }^{44-47}$. Este efecto estaría mediado por la reducción de la DHT y se asociaría a la reversión de la hipertrofia, disfunción ventricular izquierda y fibrosis ${ }^{44}$. Por otro lado, se ha visto que finasteride disminuiría el factor de crecimiento vascular endotelial, y con ello, la densidad de vasos pequeños en el tejido renal de ratas diabéticas. Sin embargo, falta verificar su 
relevancia en humanos antes de tomar conductas en relación al uso de este medicamento ${ }^{47}$.

\section{Depresión}

En la literatura existen pocos estudios en torno a este tema. El único estudio que usó métodos validados (escala de Beck) para evaluar la depresión en hombres, encontró una fuerte asociación entre la exposición a finasteride en dosis bajas y la depresión ${ }^{22}$. Se ha discutido, si estos efectos son permanentes y si es que realmente influyen en la calidad de vida, encontrándose evidencia controversial en relación a este tema ${ }^{48,49}$.

Se postula que el mecanismo, por el cual los 5-IAR podrían causar depresión, sería mediante la reducción de los niveles de neuroesteroides en el cerebro, que se ha detectado en usuarios de finasteride a dosis bajas, incluso después de su suspensión ${ }^{50,51}$. Estos neuroesteroides jugarían un rol central en la modulación de la depresión, ansiedad y estrés.

\section{Conclusión}

Finasteride ha sido amplia y exitosamente usado en el tratamiento de la HBP y AGA. Sin embargo, su uso ha reportado serios efectos adversos, principalmente de tipo sexuales y reproductivos. La evidencia disponible hasta el momento muestra que el uso de finasteride a la dosis de $1 \mathrm{mg} /$ día podría ocasionar disfunción eréctil en algunos varones. Sin embargo, no se ha demostrado que este efecto sea permanente. Por otro lado, algunos podrían ver afectada su fertilidad por uso de finasteride incluso en dosis bajas. En dicho caso, recomendamos la suspensión de este fármaco.

Finalmente, buena calidad de evidencia sugiere que finasteride no aumenta el riesgo de cáncer prostático, mientras que la literatura es controversial en cuanto al aumento de cáncer de mama.

Y por último, pareciera que la inhibición dual de las isoenzimas 5- $\alpha$ reductasas podría tener consecuencias adversas sobre ciertos procesos metabólicos, principalmente en obesos. Sin embargo, se requieren más estudios para evaluar este posible efecto deletéreo sobre ciertos parámetros de importancia clínica.

Por lo tanto, los médicos debiesen tener siem- pre presente estos eventuales efectos adversos y comentarle a sus pacientes los pro y contra de esta terapia, previo a su indicación médica.

\section{Referencias}

1. Yim E, Nole KL, Tosti A. $5 \alpha$-Reductase inhibitors in androgenetic alopecia. Curr Opin Endocrinol Diabetes Obes 2014; 21 (6): 493-8.

2. Mella JM, Perret MC, Manzotti M, Catalano HN, Guyatt G. Efficacy and safety of finasteride therapy for androgenetic alopecia: a systematic review. Arch Dermatol 2010; 146 (10): 1141-50.

3. UK MHRA. MHRA public assessment report: the risk of male breast cancer with finasteride. December 2009. MHRA: London, UK. http://www.mhra.gov.uk/home/ groups/spar/documents/websiteresources/con079340. pdf. (Accessed 9 Feb 2015).

4. McConnell JD, Roehrborn CG, Bautista OM, Andriole GL Jr, Dixon CM, et al. The long-term effect of doxazo$\sin$, finasteride, and combination therapy on the clinical progression of benign prostatic hyperplasia. N Engl J Med 2003; 349 (25): 2387-98.

5. McConnell JD, Bruskewitz R, Walsh P, Andriole G, Lieber M, Holtgrewe HL, et al. The effect of finasteride on the risk of acute urinary retention and the need for surgical treatment among men with benign prostatic hyperplasia. N Engl J Med 1998; 338 (9): 557-63.

6. Thompson IM, Goodman PJ, Tangen CM, Lucia MS, Miller GJ, Ford LG, et al. The influence of finasteride on the development of prostate cancer. N Engl J Med 2003; 349 (3): 215-24.

7. Robinson D, Garmo H, Holmberg L, Stattin P. 5- $\alpha$ reductase inhibitors, benign prostatic hyperplasia, and risk of male breast cancer. Cancer Causes Control 2015 Jun 25. [Epub ahead of print].

8. Sasco AJ, Lowenfels AB, Pasker-de Jong P. Review article: epidemiology of male breast cancer. A meta-analysis of published case-control studies and discussion of selected aetiological factors. Int J Cancer 1993; 53 (4): 538-49.

9. Thompson IM Jr, Goodman PJ, Tangen CM, Parnes HL, Minasian LM, Godley PA, et al. Long-term survival of participants in the prostate cancer prevention trial. $\mathrm{N}$ Engl J Med 2013; 369 (7): 603-10.

10. Andriole GL, Bostwick DG, Brawley OW, Gomella LG, Marberger M, Montorsi F, et al. Effect of dutasteride on the risk of prostate cancer. N Engl J Med 2010; 362 (13): 1192-202.

11. Morgentaler A, Traish AM. Shifting the paradigm of 
testosterone and prostate cancer: the saturation model and the limits of androgen-dependent growth. Eur Urol 2009; 55 (2): 310-20.

12. Traish AM, Morgentaler A. Epidermal growth factor receptor expression escapes androgen regulation in prostate cancer: a potetial molecular swith for tumour growth. Br J Cancer 2009; 101 (12): 1949-56.

13. Muller RL, Gerber L, Moreira DM, Andriole G, Castro-Santamaria R, Freedland SJ. Serum testosterone and dihydrotestosterone and prostate cancer risk in the placebo arm of the Reduction by Dutasteride Prostate Cancer Events trial. Eur Urol 2012; 62 (15): 757-64.

14. Hudson PB, Boake R, Trachtenberg J, Romas NA, Rosenblatt S, Narayan P, et al. Efficacy of finasteride is maintained in patients with benign prostatic hyperplasia treated for 5 years. The North American Finasteride Study Group. Urology 1999; 53 (4): 690-5.

15. Nickel JC, Fradet Y, Boake RC, Pommerville PJ, Perreault JP, Afridi SK, et al. Efficacy and safety of finasteride therapy for benign prostatic hyperplasia: results of a 2-year randomized controlled trial (the PROSPECT study). Proscar Safety Plus Efficacy Canadian Two year Study. CMAJ 1996; 155 (9): 1251-9.

16. Bruskewitz R, Girman CJ, Fowler J, Rigby OF, Sullivan $\mathrm{M}$, Bracken RB, et al. Effect of finasteride on bother and other health-related quality of life aspects associated with benign prostatic hyperplasia. PLESS Study Group. Proscar Long-term Efficacy and Safety Stude. Urology 1999; 54 (4): 670-8.

17. Wessells H, Roy J, Bannow J, Grayhack J, Matsumoto $\mathrm{AM}$, Tenover L, et al. Incidence and severity of sexual adverse experiences in finasteride and placebo-treated men with benign prostatic hyperplasia. Urology 2003; 61 (3): 579-84.

18. Wilt TJ, Macdonald R, Hagerty K, Schellhammer P, Tacklind J, Somerfield MR, et al. 5- $\alpha$-Reductase inhibitors for prostate cancer chemoprevention: an updated Cochrane systematic review. BJU Int 2010; 106 (10): 1444-51.

19. Mondaini N, Gontero P, Giubilei G, Lombardi G, Cai T, Gavazzi A, et al. Finasteride $5 \mathrm{mg}$ and sexual side effects: How many of these are related to a nocebo phenomenon? J Sex Med 2007; 4 (6): 1708-12.

20. Moinpour CM, Darke AK, Donaldson GW, Thompson IM Jr, Langley C, Ankerst DP, et al. Longitudinal analysis of sexual function reported by men in the Prostate Cancer Prevention Trial. J Natl Cancer Inst 2007; 99 (13): 1025-35.

21. Gupta AK, Charrette A.The efficacy and safety of $5 \alpha-$-reductase inhibitors in androgenetic alopecia: a network meta-analysis and benefit-risk assessment of finasteride and dutasteride. J Dermatolog Treat 2014; 25 (2): 156 61.

22. Belknap SM, Aslam I, Kiguradze T, Temps WH, Yarnold PR, Cashy J, et al. Adverse Event Reporting in Clinical Trials of Finasteride for Androgenic Alopecia: A Meta-analysis. JAMA Dermatol 2015; 151 (6): 600-6.

23. Glina S, Roehrborn CG, Esen A, Plekhanov A, Sorsaburu $S$, Henneges C, et al. Sexual function in men with lower urinary tract symptoms and prostatic enlargement secondary to benign prostatic hyperplasia: results of a 6-month, randomized, double-blind, placebo-controlled study of tadalafil coadministered with finasteride. J Sex Med 2015; 12 (1): 129-38.

24. Amory JK, Wang C, Swerdloff RS, Anawalt BD, Matsumoto AM, Bremner WJ, et al. The effect of 5alpha-reductase inhibition with dutasteride and finasteride on semen parameters and serum hormones in healthy men. J Clin Endocrinol Metab 2007; 92 (5): 1659-65.

25. Overstreet JW, Fuh VL, Gould J, Howards SS, Lieber MM, Hellstrom W, et al. Chronic treatment with finasteride daily does not affect spermatogenesis or semen production in young men. J Urol 1999; 162 (4): 1295 300.

26. Samplaski MK, Lo K, Grober E, Jarvi K. Finasteride use in the male infertility population: effects on semen and hormone parameters. Fertil Steril 2013; 100 (6): 1542-6.

27. Robaire B, Henderson NA. Actions of 5alpha-reductase inhibitors on the epididymis. Mol Cell Endocrinol 2006; 250 (1-2): 190-5.

28. Tu HY, Zini A. Finasteride-induced secondary infertility associated with sperm DNA damage. Fertil Steril 2011; 95 (6): 2125.

29. Richards JB, Yuan X, Geller F, Waterworth D, Bataille V, Glass D, et al. Male-pattern baldness susceptibility loci for early-onset androgenetic alopecia and their unexpected association with common diseases. PLOS Genet 2012; 8 (5): 1282-4.

30. Jacobsen SJ, Cheetham TC, Haque R, Shi JM, Loo RK. Association between 5-alpha reductase inhibition and risk of hip fracture. JAMA 2008; 300 (14): 1660-4.

31. Vestergaard P, Rejnmark L, Mosekilde L. Risk of fractures associated with treatment for benign prostate hyperplasia in men. Osteoporos Int 2011; 22 (2): 731-7.

32. Lim SY, Laengvejkal P, Panikkath R, Nugent K. The association of $\alpha$-blockers and 5 - $\alpha$ reductase inhibitors in benign prostatic hyperplasia with fractures. Am J Med Sci 2014; 347 (6): 463-71.

33. Amory JK, Anawalt BD, Matsumoto AM, Page ST, Bremner WJ, Wang C, et al. The effect of 5alpha-reductase inhibition with dutasteride and finasteride on bone mineral density, serum lipoproteins, hemoglobin, 
prostate specific antigen and sexual function in healthy young men. J Urol 2008; 179 (6): 2333-8.

34. Traish AM, Guay AT, Zitzmann M. 5 $\alpha$-Reductase inhibitors alter steroid metabolism and may contribute to insulin resistance, diabetes, metabolic syndrome and vascular disease: a medical hypothesis. Horm Mol Biol Clin Investig 2014; 20 (3): 73-80.

35. Baudrand R, Domínguez JM, Carvajal CA, Riquelme A, Campino C, Macchiavello S, et al. Over expression of hepatic $5 \alpha$-reductase and $11 \beta$-hydroxysteroid dehydrogenase type 1 in visceral adipose tissue is associated with hyperinsulinemia in morbidly obese patients. Metabolism 2011; 60 (12): 1775-80.

36. Upreti R, Hughes KA, Livingstone DE, Gray CD, Minns FC, Macfarlane DP, et al. $5 \alpha$-reductase type 1 modulates insulin sensitivity in men. J Clin Endocrinol Metab 2014; 99 (8): E1397-406.

37. Gormley GJ, Stoner E, Rittmaster RS, Gregg H, Thompson DL, Lasseter KC, et al. Effects of finasteride (MK-906), a 5 alpha-reductase inhibitor, on circulating androgens in male volunteers. J Clin Endocrinol Metab 1990; 70 (4): 1136-41.

38. Denti L, Pasolini G, Cortellini P, Sanfelici L, Benedetti $\mathrm{R}$, Cecchetti A, et al. Changes in HDL-cholesterol and lipoprotein $\mathrm{Lp}(\mathrm{a})$ after 6-month treatment with finasteride in males affected by benign prostatic hyperplasia (BPH). Atherosclerosis 2000; 152 (1): 159-66.

39. Duskova M, Hill M, Starka L. Changes of metabolic profile in men treated for androgenetic alopecia with 1 mg finasteride. Endocr Regul 2010; 44 (1): 3-8.

40. Gacci M, Corona G, Vignozzi L, Salvi M, Serni S, De Nunzio C, et al. Metabolic syndrome and benign prostatic enlargement: a systematic review and meta-analysis. BJU Int 2015; 115 (1): 24-31.

41. Loke YK, Ho R, Smith M, Wong O, Sandhu M, Sage W, et al. Systematic review evaluating cardiovascular events of the 5-alpha reductase inhibitor-Dutasteride. J Clin Pharm Ther 2013; 38 (5): 405-15.

42. Hsieh TF, Yang YW, Lee SS, Lin TH, Liu HH, Tsai $\mathrm{TH}$, et al. Use of 5-alpha-reductase inhibitors did not increase the risk of cardiovascular diseases in patients with benign prostate hyperplasia: a five-year follow-up study. PLoS One 2015; 10 (3): e0119694.
43. Souverein PC, Herings RM, Man in 't Veld AJ, de la Rosette JJ, Farmer RD, Leufkens HG. Study of the association between ischemic heart disease and use of alpha-blockers and finasteride indicated for the treatment of benign prostatic hyperplasia. Eur Urol 2002; 42 (3): 254-61.

44. Zwadlo C, Schmidtmann E, Szaroszyk M, Kattih B, Froese N, Hinz H, et al. Antiandrogenic therapy with finasteride attenuates cardiac hypertrophy and left ventricular dysfunction. Circulation 2015; 131 (12): 1071-81.

45. Chung CC, Hsu RC, Kao YH, Liou JP, Lu YY, Chen YJ. Androgen attenuates cardiac fibroblasts activations through modulations of transforming growth factorand angiotensin II signaling. Int J Cardiol 2014; 176 (2): 386-93.

46. Rubio-Gayosso I, Ramírez-Sánchez I, Ita-Islas I, Ortiz-Vilchis P, Gutiérrez-Salmean G, Meaney A, et al. Testosterone metabolites mediate its effects on myocardial damage induced by ischemia/reperfusion in male Wistar rats. Steroids 2013; 78 (3): 362-9.

47. Tian HL, Zhao CX, Wu HY, Xu ZX, Wei LS, Zhao RT, et al. Finasteride reduces microvessel density and expression of vascular endothelial growth factor in renal tissue of diabetic rats. Am J Med Sci 2015; 349 (6): 516-20.

48. Irwig MS. Depressive symptoms and suicidal thoughts among former users of finasteride with persistent sexual side effects. J Clin Psychiatry 2012; 73 (9): 1220-3.

49. Rahim-Ardabili B, Pourandarjani R, Habibollahi P, Mualeki A. Finasteride induced depression: a prospective study. BMC Clin Pharmacol 2006; 6: 7.

50. Melcagni RC, Caruso D, Abbiati F, Giati S, Calabrese D, Piazza F, et al. Neuroactive steroid levels are modified in cerebrospinal fluid and plasma of post-finasteride patients showing persistent sexual side effects and anxious/ deppressive symptomatology. J Sex Med 2013; 10 (10): 2598-603.

51. Caruso D, Abbiati F, Giatti S, Romano S, Fusco L, Cavaletti G, et al. Patients treated for male pattern hair with finasteride show, after discontinuation of the drug, altered levels of neuroactive steroids in cerebrospinal fluid and plasma. J Steroid Biochem Mol Biol 2015; 146: 74-9. 